\title{
线性复杂度曲线可控序列 ${ }^{*}$
}

\author{
林须端蔡长年 \\ (北京邮电学院电信工程系)
}

关链词随序列、序列密码、信息论

\section{一、记号说明}

设 $S-\left(S_{0}, S_{1}, S_{2}, \cdots\right)$ 为有限域 $G F(q)$ 上的无穷序列, $S^{n}-\left(S_{0}, s_{1}, \cdots, S_{x-1}\right)$, 序 列 $S^{n}$ 的线性复杂度 $L_{n}(S)=\min \left\{l: S_{i}=-\sum_{i=1}^{l} C_{i} S_{i-i}, i=l, l+1, \cdots, n-1, C_{1}, C_{2}\right.$, $\left.\cdots, C_{1} \in G F(q)\right\}$, 序列的线性复杂度曲线为 $L=\left(L_{0}(S), L_{1}(S), L_{2}(S), \cdots\right)$. 由序列的 随机性与复杂度关系可知, 适合作为序列密码密钥的伪随机序列, 其线性复杂度曲线应接近于 $\frac{N}{2}$ 这条直线 ${ }^{[1-3]}$. 本文提出了产生具有好的线性复杂度曲线及分量分布均匀的序列的算法, 并研究了这类序列的有关性质.

\section{二、算法及序列性质}

令 $C^{(N)}(D)-1+C_{i}^{(N)} D+\cdots+C_{L_{N}^{(N)}}^{(S)} D^{L_{N}(S)}$ 为 $S^{N}$ 的最短线性 反馈移位寄存器 (linear feedback shift register, LFSR) 的联接多项式,

$$
d_{N}-s_{N}+\sum_{i=1}^{L_{N}(s)} C_{i}^{(N)} S_{N-i}
$$

由 Massey 的结果 ${ }^{[4]}$ 可知:

若 $d_{N}=0$, 则

$$
\begin{aligned}
& L_{N+1}(S)-L_{N}(S), \\
& C^{(N+1)}(D)-C^{(N)}(D) ;
\end{aligned}
$$

若 $d_{N} \neq 0$, 则

$$
L_{N+1}(S)=\left\{\begin{array}{l}
L_{N}(S), \text { 当 } L_{N}(S) \geqslant \frac{N+1}{2} \text { 时, } \\
N+1-L_{N}(S), \text { 当 } L_{N}(S)<\frac{N+1}{2} \text { 时, }
\end{array}\right.
$$

且 $C^{(N+1)}(D)=C^{(N)}(D)-d_{N} d_{m}^{-1} D^{N-m} C^{(m)}(D)$, 其中 $m$ 为满足 $L_{m}(S)<L_{N}(S)-L_{m+1}(S)$ 的整数.

本文1989年 4 月 14 日收到. 1989 年 8 月 11 日收到修改稀.

- 国家自然科学基金资助项目. 
因此我们可以通过序列 $d-\left(d_{0}, d_{1}, \cdots\right)$ 的选择来产生序列 $S$, 并控制 $S$ 的线性复杂度 曲线和分量取值分布, 使之能满足密钥序列的要求. 算法如下:

1) 初始化: $N \leftarrow 0, C(D) \leftarrow 1, L \leftarrow 0, B(D) \leftarrow 1, x \leftarrow 1, b \leftarrow 1, n_{t_{1}}=n_{f_{1}}-\cdots=$ $n_{t_{\eta-1}}-0$, 其中 $f_{0}, \cdots, f_{q-1}$ 为 $G F(q)$ 的不同元素, 给定 $E, K, n$.

2) 若 $N-n$, 停止,否则

3) 若 $L \geqslant \frac{N+1}{2}$, 置 $F-0$, 转选 $d$ 子程序, 计算 $S_{N}-d-\sum_{i=1}^{L} C_{i} S_{N-i}$, $C(D) \leftarrow C(D)-d b^{-1} D^{x} B(D), x \leftarrow x+1$ 转到 6$)$.

4) 若 $L<\frac{N+1}{2}$, 当 $L \geqslant \frac{N}{2}-E+1$ 时, $F-0$, 转选 $d$ 子程序:

若 $d-0$, 计算 $S_{N}--\sum_{i=1}^{L} C_{i} S_{N-i}, x \leftarrow x+1$, 转到 6);

若 $d \neq 0$, 转到 5 ). 当 $\frac{N+1}{2}-E \leqslant L<\frac{N}{2}-E+1$ 时, $F-1$, 转选 $d$ 子程序.

5) 计算 $S_{N}-d-\sum_{i=1}^{L} C_{i} S_{N-i}, L \leftarrow N+1-L, T(D) \leftarrow C(D), C(D) \leftarrow C(D)-$ $d b^{-1} D^{x} B(D), B(D) \leftarrow T(D), x-1, b-d$.

6) 若 $S_{N}-f_{i}, n_{t_{i}} \leftarrow n_{t_{i}}+1, N \leftarrow N+1$, 转到 2).

选 $d$ 子程序:

(1) 将 $n_{t_{0}}, n_{t_{1}}, \cdots, n_{t_{q-1}}$ 从小到大排列为 $n_{i_{i_{0}}}, n_{t_{i_{1}}}, \cdots, n_{i_{i_{q-1}}}$.

(2) 若 $F-1$, 从 $\left\{f_{i_{0}}, \cdots, f_{i_{K}}\right\}-\left\{-\sum_{i=1}^{L} c_{i} s_{N-i}\right\}$ 中任取一元素 $f$;

若 $F-0$, 从 $\left\{f_{i_{0}}, \cdots, f_{i k}\right\}$ 中取一元素 $f$.

（3）计算 $d-\sum_{i=1}^{L} c_{i} s_{N_{-i}}+t$, 返回主程序.

$L_{0}(S)-0$, 当 $L_{N}(S) \geqslant \frac{N+1}{2}$ 时, 若有 $\left|L_{N}(S)-\frac{N}{2}\right| \leqslant E$, 则对任意的 $d_{N}$, 有 $\left|L_{N+1}(S)-\frac{N+1}{2}\right|-\left|L_{N}(S)-\frac{N}{2}\right|-\frac{1}{2} \leqslant E-\frac{1}{2}$; 当 $L_{N}(S)<\frac{N+1}{2}$ 时, 由算法, $\left|\frac{N+1}{2}-L_{N}(S)\right| \leqslant E-\frac{1}{2}, d_{N}$ 可任意取值, 则 $\left|L_{N+1}(S)-\frac{N+1}{2}\right|-\mid \frac{N+1}{2}-$ $L_{N}(S) \mid \leqslant E-\frac{1}{2}$; 由 $\frac{N+1}{2}-E \leqslant L_{N}<\frac{N}{2}-E+1, d_{N} \neq 0$, 则 $\left|L_{N+1}(S)-\frac{N+1}{2}\right|=$ $\left|L_{N}(S)-\frac{N+1}{2}\right| \leqslant E$. 综上所得

栍质 1 由上述算法产生的所有序列的线性复杂度满足 $\left|L_{N}(S)-\frac{N}{2}\right| \leqslant E, N-0$, $1,2, \cdots$. 
由算法可知, $d-\left(d_{0}, d_{1}, d_{2}, \cdots\right)$ 与 $S-\left(S_{0}, S_{1}, S_{2}, \cdots\right)$ 是一一对应的, 且曲线 $L_{N}(S)$ 是关于直线 $\frac{N}{2}$ 对称, 再由 $d_{N}$ 的取值与 $L_{N+1}(S)$ 的关系, 算法中去掉 $K$ 的限制或 $K>q$, 则 有

性质 2 具有给定线性复杂度曲线 $L^{n+1}=\left(L_{0}(S), L_{1}(S), \cdots, L_{n}(S)\right)$, 且 $\left|L_{N}(S)-\frac{N}{2}\right| \leqslant E, N=0,1, \cdots, n$, 的 $G F(q)$ 上序列总数为

$q^{\frac{n^{\prime}}{2}} \cdot(q-1)^{\prime} \cdot R$, 其中 $n^{\prime}$ 为 $L^{n+1}$ 最后一个落在直线 $\frac{N}{2}$ 上的点, $J$ 为 $L^{n+1}$ 中的跃 点数, $R$ 满足: $1 \leqslant R \leqslant q^{\left\lfloor\frac{n-n^{\prime}}{2}\right\rfloor} \leqslant q^{E}$.

由性质 2 可见, 略去 $R$ 不计, 满足给定线性复杂度曲线的序列随着跃点数的增大而增多. 当 $L_{N}(S)=\left\lfloor\frac{N+1}{2}\right\rfloor$ 时, 即 $S^{n}$ 为完备的线性复杂度序列, 其总数为 $q^{\left\lfloor\frac{\pi}{2}\right\rfloor} \cdot(q-1)^{\left\lfloor\frac{n+1}{2}\right\rfloor}$.

经过类似的分析,算法中不加 $K 、 E$ 的限制, 可得以下性质:

性质 $3 G F(q)$ 上满足线性复杂度曲线 $L^{n+1}$ 中有 $J$ 个跃点且 $L_{n}(S)-\frac{n}{2}$ 的序列 $S^{n}$ 的数目为 $\left(\begin{array}{c}\frac{n}{2}-1 \\ J-1\end{array}\right) \cdot q^{\frac{\pi}{2}} \cdot(q-1)^{J}$.

\section{三、总结}

由上述算法产生的序列 $S^{n}$, 其分量取值的分布随 $K$ 的选择而受控制, 当 $n$ 很大时, 分布 将是概率 1 均匀的. 但对序列伪随机性的限制条件不能太多、太具体, $K$ 和 $E$ 的大小应按实际 需要确定 ${ }^{[1]}$.

基于 Berlekamp-Massey 算法 ${ }^{[1]}$, 我们提出的算法可用于产生有好的线性复杂度曲线及 分量分布、适合序列密码要求的序列, 且同时可得产生对应序列的 LFSR. 这些结果和有关序 列性质的研究对密码的分析和设计都是很有价值的.

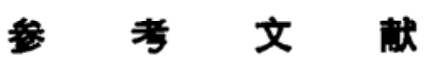

[1] Kolmogorov, A. N., IEEE Trans. Injor. Theor., IT-14(1968), 5: 662-664.

[2] Rueppel, R. A., Analysis and Design of Stream Ciphers, Springer-Verlag, Berlin, 1986.

[3] Piper, F. C., Elektrotech. Masch. Bau., 104(1987), 12: 564-568.

[4] Massey, J. L., IEEE Trans. Infor. Theor., IT-15(1969), 1:122-127. 